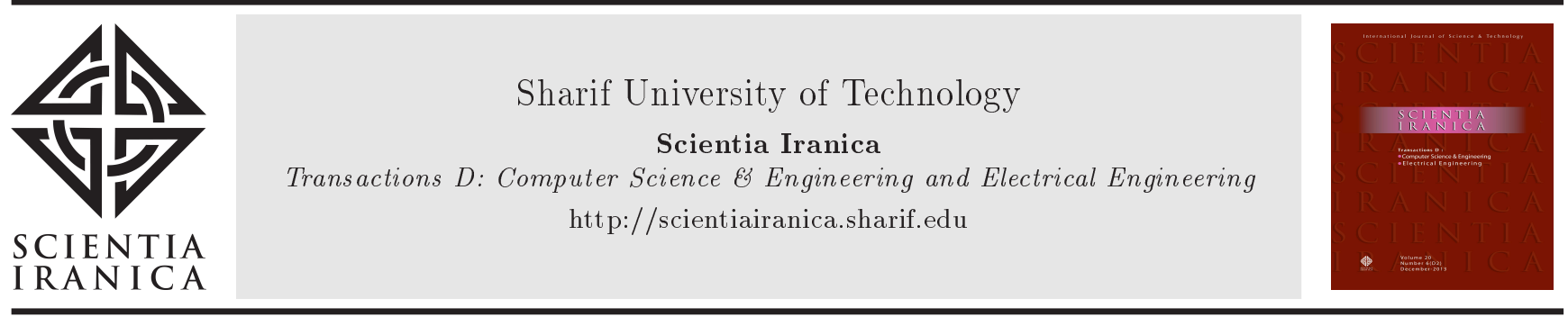

\title{
Evaluating the effect of SSSC stabilizer in different control channels on sub-synchronous resonance oscillations
}

\author{
M.R. Shakarami ${ }^{a, *}$, A. Asadi Ghyasvand ${ }^{\mathrm{a}}$, and A. Kazemi ${ }^{\mathrm{b}}$ \\ a. Department of Engineering, Lorestan University, Lorestan, P.O. Box 68151-44316, Iran. \\ b. Department of Electrical Engineering, Iran University of Science and Technology, Tehran, Iran. \\ Received 4 August 2015; received in revised form 20 August 2016; accepted 26 September 2016
}

\author{
KEYWORDS \\ Eigenvalues; \\ Static Synchronous \\ Series Compensator \\ (SSSC); \\ Sub-Synchronous \\ Resonance (SSR); \\ Gray Wolves \\ Optimization (GWO) \\ algorithm.
}

\begin{abstract}
To increase transmitted power, series capacitors may be used in the transmission lines. A compensated transmission line together with a turbine-generator may cause Sub-Synchronous Resonance (SSR) phenomena in a power system. In this paper, details of dynamic modeling of a single machine power system connected to the network through a transmission line, compensated with a series capacitor, have been presented. Static Synchronous Series Compensator (SSSC) as one of series FACTS devices has been used for the damping of the SSR oscillations. For this purpose, a linear damping SSSCbased stabilizer with lead-lag structure is incorporated into SSSC controller. To determine parameters of SSSC stabilizer, a new multi-objective function has been proposed. In this function, in addition to improving stability of critical modes, the gain of the stabilizer has been considered. To have a stabilizer with a minimum-phase structure, suitable constraints have been determined and added to the objective function. To optimize the objective function, a recently developed optimization technique known as Grey Wolf Optimization (GWO) algorithm has been applied. The eigenvalues analysis and nonlinear simulations of the IEEE First Benchmark Model (FBM) power system with a SSSC stabilizer illustrate the effectiveness of the proposed method in suppressing SSR oscillations in the studied system.
\end{abstract}

(C) 2018 Sharif University of Technology. All rights reserved.

\section{Introduction}

Series capacitors have been successfully used for many years in order to enhance the stability and increase the power transfer capability of the high-voltage transmission networks [1]. However, when this technique is applied together with a turbine-generator, it may lead to Sub-Synchronous Resonance (SSR) oscillations. SSR problem results from the interactions between electric

*. Corresponding author. Tel.: +98663 3120086;

Fax: +986633120086

E-mail address: Shakarami.mr@lu.ac.ir (M.R. Shakarami)

doi: $10.24200 /$ sci.2017.4364 modes in the network and mechanical modes in turbine shaft, which disturb dynamic stability of the system and probably destroy the generator [2]. Nowadays, the use of wind power generations is increasing noticeably. SSR phenomena with induction generators in wind farms connected to the network through compensated transmission lines may occur [3].

To damp SSR oscillations, different techniques have been presented in the literature. In [4], effect of the gain of Automatic Voltage Regulator (AVR) on bifurcations of SSR in a power system has been studied. The results show that the locations of HOPF bifurcations can be controlled by the gain value of the AVR. A supplementary damping controller for generator excitation system to mitigate SSR oscillations 
has been proposed in [5]. In [6], a Gat Controlled Series Capacitor (GCSC) has been used to damp SSR oscillations. In $[7,8]$, fuzzy logic and PSO algorithms have been used to determine parameters of a supplementary damper (DSSC) for a DFIG to reduce SSR oscillations in a wind farm turbine. A novel auxiliary damping stabilizer to mitigate SSR using active power control of a DFIG-based wind farm has been presented in [9]. In this method, the genetic algorithm determines the parameters of the PID damping controller. The SSR phenomenon in the presence of Type- 2 of wind turbines has been studied in [10]. In [11], the HOPF bifurcation of the equilibrium points and their corresponding limit cycles have been applied to the study of sub-synchronous interaction between wind farms and series-compensated transmission lines. In [12], a novel two-Degree-Of-Freedom (2DOF) control strategy combined with a damping control loop is designed and analyzed to enhance the system stability and alleviate the SSR that may arise due to the induction generator effect. In [13], a PI damping controller based on Wide-Area Measurement System (WAMS) has been designed and added to the main loop of PV plat to damp SSR oscillations. A bypass-damping filter can be a countermeasure to increase damping of SSR oscillations [14]. In addition, it can help reduce transient overvoltage of the series capacitor in the series-compensated systems. In [15], a global optimal control design to guarantee tensional stability under all possible operation conditions has been proposed. In this method, parameters of multiple excitation systems are simultaneously determined to damp SSR oscillations. In [16], a methodology for the evaluation of risk of SSR in meshed compensated AC networks has been proposed. In this method, severity of the SSR phenomena in different network configurations caused by fixed series compensation and probabilistic behavior of the power system, which is due to random outage of lines, has been evaluated. The FACTS devices are such that they can rapidly control various network operating conditions; this capability of these devices can improve the stability of the power systems [17]. To increase damping of SSR oscillations, FACTS devices are widely utilized. These devices should be enhanced with an auxiliary damping stabilizer to provide extra damping characteristic. Design of a SSSC stabilizer to damp SSR oscillations has been analyzed in [18].

In [19], effect of SVC and TCSC controllers on mitigating SSR oscillations caused by an induction generator has been studied. Coordinated design of Static VAR Compensators (SVCs) and Power System Stabilizers (PSSs) to damp SSR oscillations has been investigated in [20]. In [21], an auxiliary STATCOM-based stabilizer to damp SSR oscillations has been proposed. In [22], the use of TCSC-based stabilizer to control bifurcation of the SSR in a multi-machine power system has been investigated. The results showed that by adding a TCSC-based stabilizer, all bifurcations could be eliminated. In [23], Real-Time Recurrent Learning (RTRL) algorithm, in which the Neural Network (NN) is trained in real time, is applied to the design of TCSCbased stabilizer to damp SSR oscillations. A procedure for auxiliary Sub-Synchronous Damping Controller (SSDC) on STATCOM based on nonlinear optimization to meet the specifications of the damping torque in the range of critical torsional frequencies has been proposed in [24]. In [25], design of a SSSC-based stabilizer to damp sub-synchronous oscillations is presented as an optimization problem. To solve the problem, Gravitational Search Algorithm (GSA) has been applied. In this reference, the Integral-Time-Absolute-Error (ITAE) is considered as a performance index. In [26], a methodology for selecting an optimal combination of Thyristor-Controlled Series Capacitors (TCSC) and fixed series capacitors for compensation of transmission lines based on minimization of the risk of exposure to SSR has been proposed. In [27], a TCSC was utilized to reduce SSR oscillations using a supplementary controller. SSSC can control the transmitted power by transforming inductive states into capacitive ones [28]. Furthermore, by adding a Supplementary Damping Controller (SDC) to a SSSC, low-frequency oscillations can be dampened in power systems [29]. To implement a stabilizer in a SSSC, phase and magnitude controller channels can be utilized. In [30,31], SSSC's dampening capability of controlling SSR is discussed in detail. In these references, a supplementary damping controller is added to the primary control loop, and damper's parameters are determined by evolutionary algorithms.

Most of the above research studies have considered an objective function only including critical eigenvalues to improve SSR oscillations, while, a suitable stabilizer, in addition to improving stability of the system, must have a low control cost and a good response time characteristic. On the other hand, some FACTS devices, such as SSSC and STATCOM, which have been usually applied to improve damping of the SSR oscillations, have two control channels known as phase and magnitude control channels. The stabilizer in one of these control channels may be more effective in improving stability of oscillations in a power system.

In [28], the effect of a SSSC stabilizer in different control channels on inter-area oscillations was studied. The results showed that SSSC stabilizer in the phase control channel was more effective in damping interarea oscillations than the magnitude control channel. However, the effect of a SSSC stabilizer in different control channels on SSR oscillations has not been well studied in the reported literatures.

In this paper, the model of a single machine sixmass power system compensated by a capacitor and together with a SSSC stabilizer is presented to study 


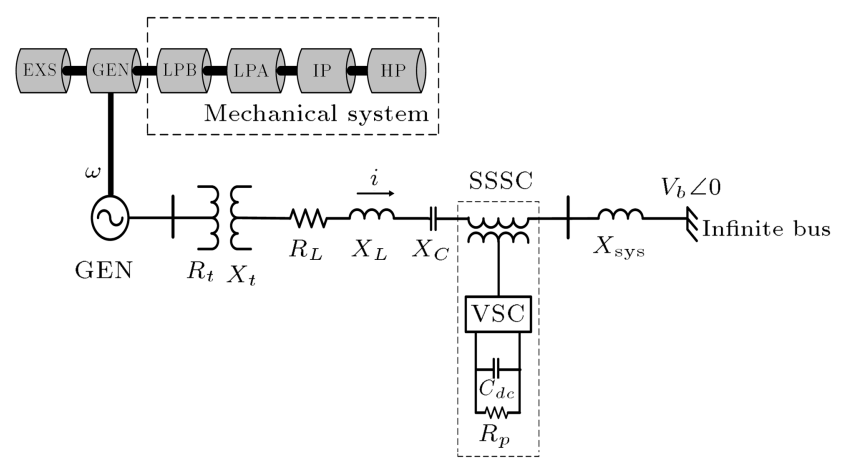

Figure 1. The modified IEEE first benchmark model with a SSSC.

SSR. To improve stability of the system, a lead-lag stabilizer has been considered. The stability of SSR oscillations is presented as a new objective function. To have a stabilizer with minimum control cost, the gain of the stabilizer is considered in the objective function. In addition, to have a SSSC stabilizer with minimum phase structure, the suitable constraints have been calculated and added to the objective function. The Grey Wolf Optimization algorithm (GWO) is used to optimize the objective function. The effect of a SSSC stabilizer in different control channels on SSR oscillations has been studied.

\section{Power system modeling}

In order to study sub-synchronous resonance phenomenon, the IEEE First Benchmark Model (FBM) with a SSSC was used. This model is shown in Figure 1. Parameters of the system were adopted from [32]. This system has been composed of a generator (model 2.2), a multi-mass (multi-stage) turbine, a governor, an exciter, and a compensated transmission line with a series capacitor and a SSSC. Each part was modeled autonomously and then combined to make the power system.

The above system was studied based on the following assumptions:

1. The power produced by generator, flowing toward the load, is $S=0.9-j 0.392 \mathrm{p} . \mathrm{u}$;

2. Mechanical power input to turbine is constant;

3. Relation between flux and current in the iron portions is supposed to be linear.

\subsection{Static Synchronous Series Compensator $(S S S C)$}

Voltage source for a SSSC is a DC source. This voltage source is connected to an $\mathrm{AC}$ network by a converter. The DC voltage is converted into ac voltage according to the switching type and angle [33]. A general structure of a SSSC is shown in Figure 2.

Here, $R_{s}$ and $X_{s}$ are series resistance and reactance of a SSSC, respectively. $I_{L}$ is the current passing

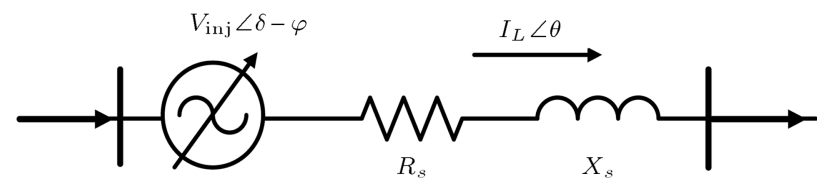

Figure 2. Schematic of a SSSC.

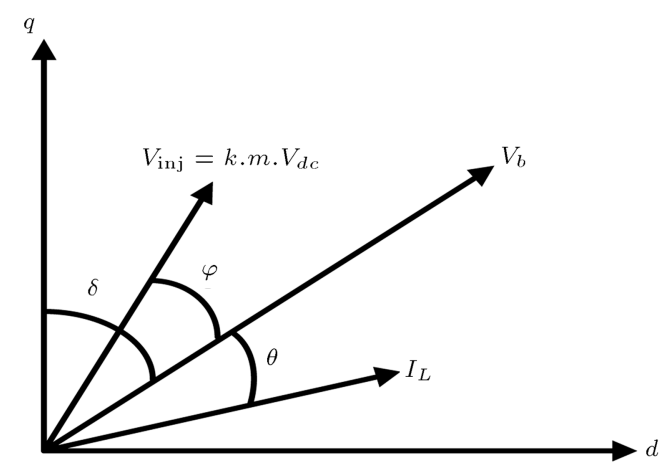

Figure 3. Phasor diagram for voltage and current of the transmission line

through transmission line and $V_{\text {inj }}$ is the injected voltage by the SSSC. The phasor diagram for the transmission line in $d q$ basis is shown in Figure 3.

The injected voltage is as follows:

$$
\overline{V_{\mathrm{inj}}}=V_{\mathrm{inj} d}+j V_{\mathrm{inj} q}=\left|V_{\mathrm{inj}}\right| \angle(\delta-\varphi),
$$

where $\varphi$ is the angle of the injected voltage by the SSSC, and $\delta$ is the torque angle. $V_{\operatorname{inj} d}$ and $V_{\operatorname{inj} q}$ are the injection voltages by the SSSC in $d q$ basis, which are obtained as follows:

$$
\begin{aligned}
& V_{\mathrm{inj} d}=k \cdot m \cdot V_{d c} \sin (\delta-\varphi), \\
& V_{\mathrm{inj} q}=k \cdot m \cdot V_{d c} \cos (\delta-\varphi) .
\end{aligned}
$$

Transmission line current in $d q$ basis is written as follows:

$$
\overline{I_{L}}=i_{d}+j i_{q}=\left|I_{L}\right| \angle \theta
$$

where $\theta$ is the angle between voltage and current in the infinite bus. Also, $i_{d}$ and $i_{q}$ are $d$ and $q$ components of line currents, respectively.

\subsection{Generator and transmission line}

A series compensated long transmission line with a $\mathrm{SSSC}$ is shown in Figure 4.

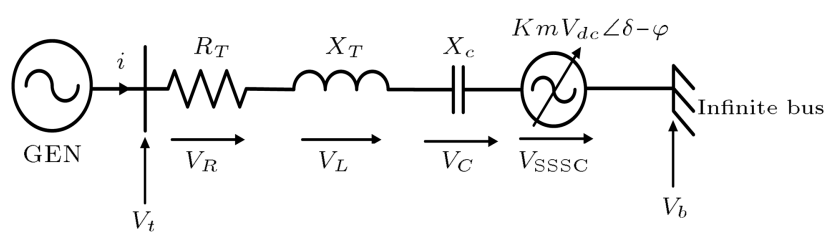

Figure 4. Schematic representation of IEEE FBM with a SSSC. 
Here, $X_{T}$ and $R_{T}$ are the equivalent reactance and impedance of the transmission line, respectively, and $V_{t}$ is the value of generator's terminal voltage. Stator equations in per-unit basis are considered as follows [2]:

$$
\begin{aligned}
& \frac{1}{\omega_{0}} \frac{d \psi_{d}}{d t}=R_{a}^{*} i_{d}+\frac{\omega}{\omega_{0}} * \psi_{q}+V_{d}, \\
& \frac{1}{\omega_{0}} \frac{d \psi_{q}}{d t}=R_{a}^{*} i_{q}-\frac{\omega}{\omega_{0}} * \psi_{d}+V_{q} .
\end{aligned}
$$

$R_{a}$ represents resistance in stator windings, $V_{q}$ and $V_{d}$ are generator's terminal voltages in $d q$ basis, $\omega_{0}$ and $\omega$ are rotor speed synchronous and rotor speed of generator, respectively, $i_{q}$ and $i_{d}$ are currents in stator windings which flow into transmission line, and $\psi_{d}$ and $\psi_{q}$ are fluxes of the stator whose equations are as follows:

$$
\begin{aligned}
& \psi_{d}=-L_{d}^{*} i_{d}+L_{a d}^{*} i_{f d}+L_{a d}^{*} i_{1 d}, \\
& \psi_{q}=-L_{q}^{*} i_{q}+L_{a q}^{*} i_{1 q}+L_{a q}^{*} i_{2 q}
\end{aligned}
$$

where $L_{d}$ and $L_{q}$ are synchronous inductances in the $d-q$ reference frame, and $L_{a d}$ and $L_{a q}$ are the mutualinductances. Equations for rotor are considered as follows [2]:

$$
\begin{aligned}
& \frac{1}{\omega_{0}} \frac{d \psi_{f d}}{d t}=-R_{f d}^{*} i_{f d}+e_{f d}, \\
& \frac{1}{\omega_{0}} \frac{d \psi_{1 d}}{d t}=-R_{1 d}^{*} i_{1 d}, \\
& \frac{1}{\omega_{0}} \frac{d \psi_{1 q}}{d t}=-R_{1 q}^{*} i_{1 q}, \\
& \frac{1}{\omega_{0}} \frac{d \psi_{2 q}}{d t}=-R_{2 q}^{*} i_{2 q},
\end{aligned}
$$

where $R_{2 q}, R_{1 q}, R_{1 d}$, and $R_{f d}$ are winding resistances, and $i_{2 q}, i_{1 q}, i_{1 d}$, and $i_{f d}$ are currents through the rotor's windings. $e_{f d}$ is the voltage source of excitation winding and $\psi_{2 q}, \psi_{1 q}, \psi_{1 d}$, and $\psi_{f d}$ are fluxes of windings of rotor whose equations are as follows [2]:

$$
\begin{aligned}
& \psi_{f d}=L_{f f d}{ }^{*} i_{f d}+L_{a d}{ }^{*} i_{1 d}-L_{a d}{ }^{*} i_{d}, \\
& \psi_{1 q}=L_{11 q}{ }^{*} i_{1 q}+L_{a q}{ }^{*} i_{2 q}-L_{a q}{ }^{*} i_{q}, \\
& \psi_{1 d}=L_{a d}{ }^{*} i_{f d}+L_{11 d}{ }^{*} i_{f d}-L_{a d}{ }^{*} i_{d}, \\
& \psi_{2 q}=L_{a q}{ }^{*} i_{1 q}+L_{22 q}{ }^{*} i_{2 q}-L_{a q}{ }^{*} i_{q},
\end{aligned}
$$

where $L_{f f d}$ and $L_{11 d}$ are self-inductances of rotor windings, and $L_{11 q}$ and $L_{22 q}$ are self-inductances of damper windings. Equations for the series capacitor in $d_{q}$ basis are considered as follows:

$$
\begin{aligned}
& \frac{d V_{c d}}{d t}=\omega^{*} V_{c q}+\omega_{0}^{*} X_{c}^{*} i_{d}, \\
& \frac{d V_{c q}}{d t}=-\omega^{*} V_{c d}+\omega_{0}^{*} X_{c}^{*} i_{q},
\end{aligned}
$$

where $V_{c q}$ and $V_{c d}$ are the voltages in $d q$ basis, $X_{C}$ is reactance for the series capacitor in the transmission line. By considering Figure 4 and simple calculations, the vector of state variables for electric side of the system can be calculated as follows:

$$
\begin{aligned}
& {\left[\frac{d x_{\text {Elec }}}{d t}\right]=\left[\begin{array}{lllll}
\frac{d i_{d}}{d t} & \frac{d i_{q}}{d t} & \frac{d i_{f d}}{d t} & \frac{d i_{1 q}}{d t} & \frac{d i_{1 d}}{d t}
\end{array}\right.} \\
& \left.\frac{d i_{2 q}}{d t} \quad \frac{d V_{c d}}{d t} \frac{d V_{c q}}{d t}\right]^{T} .
\end{aligned}
$$

\subsection{Mechanical side and exciter}

Mechanical side of the generator, shown in Figure 5 [34], is composed of a High Pressure (HP) turbine, an Intermediate Pressure (IP) turbine, two low pressure (LPA and LPB) turbines, a rotor, and an excitation system. Combination of the masses forms a six-mass system.

\subsubsection{Turbines}

Equations related to the turbines are as follows:

$$
\begin{aligned}
\frac{d \delta_{H}}{d t}= & \omega_{H}-\omega_{0} \\
\frac{d \omega_{H}}{d t}= & \omega_{0} \frac{K_{I H}}{M_{H}} \delta_{I}-\omega_{0} \frac{K_{I H}}{M_{H}} \delta_{H}+\frac{D_{H}}{M_{H}}\left(\omega_{0}-\omega_{H}\right) \\
& +\frac{\omega_{0}^{2}}{M_{H}{ }^{*} \omega_{H}} P_{H} \\
\frac{d \delta_{I}}{d t}= & \omega_{I}-\omega_{0}, \\
\frac{d \omega_{I}}{d t}= & \omega_{0} \frac{K_{A I}}{M_{I}} \delta_{A}-\omega_{0} \frac{K_{A I}+K_{I H}}{M_{I}} \delta_{I}+\omega_{0} \frac{K_{I H}}{M_{I}} \delta_{H} \\
& +\frac{D_{I}}{M_{I}}\left(\omega_{0}-\omega_{I}\right)+\frac{\omega_{0}^{2}}{M_{I}^{*} \omega_{I}} P_{I} \\
\frac{d \delta_{A}}{d t}= & \omega_{A}-\omega_{0},
\end{aligned}
$$

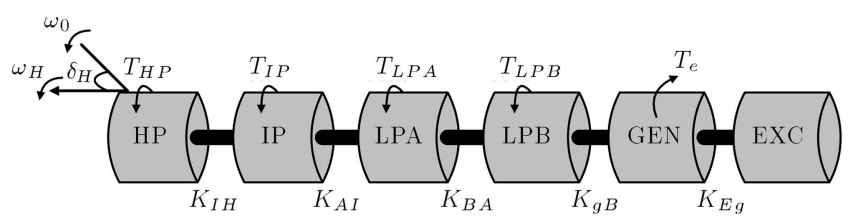

Figure 5. Structure of a typical six-mass shaft system model. 


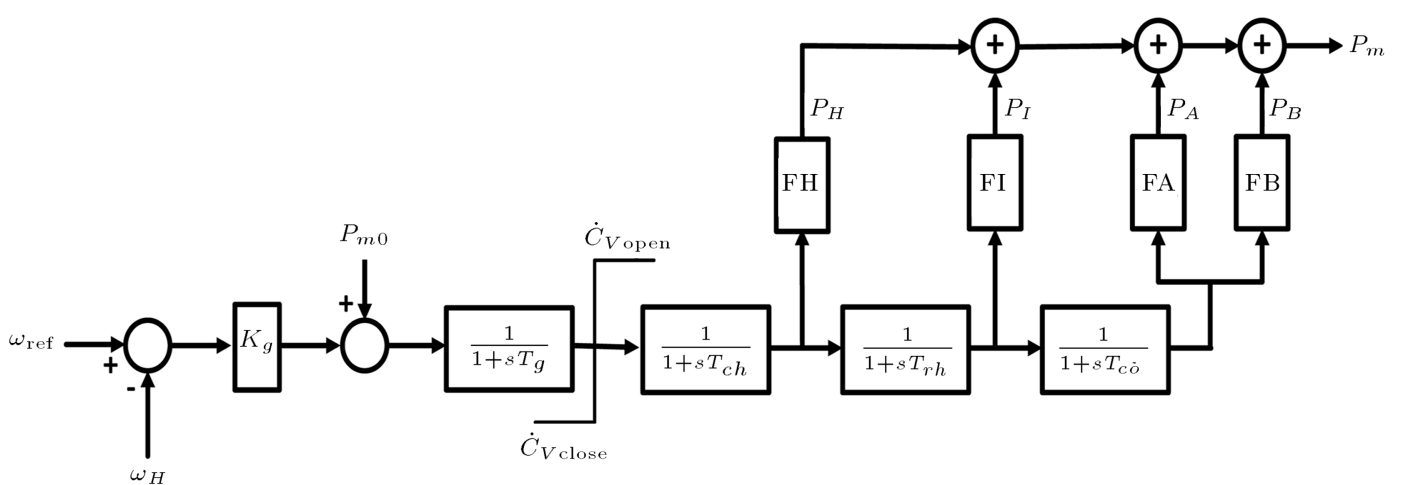

Figure 6. Block diagram of the governor and turbine.

$$
\begin{aligned}
\frac{d \omega_{A}}{d t}= & \omega_{0} \frac{K_{B A}}{M_{A}} \delta_{B}-\omega_{0} \frac{K_{B A}+K_{A I}}{M_{A}} \delta_{A}+\omega_{0} \frac{K_{A I}}{M_{A}} \delta_{I} \\
& +\frac{D_{A}}{M_{A}}\left(\omega_{0}-\omega_{A}\right)+\frac{\omega_{0}^{2}}{M_{A}{ }^{*} \omega_{A}} P_{A} \\
\frac{d \delta_{B}}{d t}= & \omega_{B}-\omega_{0}, \\
\frac{d \omega_{B}}{d t}= & \omega_{0} \frac{K_{g B}}{M_{B}} \delta-\omega_{0} \frac{K_{g B}+K_{B A}}{M_{B}} \delta_{B}+\omega_{0} \frac{K_{B A}}{M_{B}} \delta_{A} \\
& +\frac{D_{B}}{M_{B}}\left(\omega_{0}-\omega_{B}\right)+\frac{\omega_{0}^{2}}{M_{B}{ }^{*} \omega_{B}} P_{B}
\end{aligned}
$$

$\frac{d \delta}{d t}=\omega-\omega_{0}$

$$
\begin{aligned}
\frac{d \omega}{d t}= & \omega_{0} \frac{K_{E g}}{M_{g}} \delta_{E}-\omega_{0} \frac{K_{E g}+K_{g B}}{M_{g}} \delta+\omega_{0} \frac{K_{g B}}{M_{g}} \delta_{B} \\
& +\frac{D_{g}}{M_{g}}\left(\omega_{0}-\omega\right)-\frac{D_{g}}{M_{g}} T_{e},
\end{aligned}
$$

$$
\begin{aligned}
& \frac{d \delta_{E}}{d t}=\omega_{E}-\omega_{0}, \\
& \frac{d \omega_{E}}{d t}=-\omega_{0} \frac{K_{E g}}{M_{E}} \delta_{E}+\omega_{0} \frac{K_{E g}}{M_{E}} \delta+\frac{D_{E}}{M_{E}}\left(\omega_{0}-\omega_{E}\right),
\end{aligned}
$$

where $\delta_{H}, \delta_{I}, \delta_{A}, \delta_{B}$, and $\delta_{E}$ are rotor angles of high-pressure turbine, intermediate-pressure turbine, two low-pressure turbines, and rotor angle of exciter turbine, respectively. $\omega_{H}, \omega_{I}, \omega_{A}, \omega_{B}, \omega$, and $\omega_{E}$ are angular velocities for each shaft section. Here, $D$ is damping coefficient, $M$ is inertia constant in seconds, $k$ is the stiffness for each shaft section, and $T_{e}$ is output torque of synchronous machine as follows:

$$
T_{e}=\psi_{d}^{*} i_{q}-\psi_{q}^{*} i_{d}
$$

\subsubsection{Governor}

The block diagram of the four-stage turbine and the associated electro-hydraulic governor [35] are shown in
Figure 6. The corresponding dynamic equations based on the block diagram are as follows:

$$
\begin{aligned}
\frac{d C_{V}}{d t} & =-\frac{1}{T_{g}} C_{V}+\frac{1}{T_{g}} P_{m 0}-\frac{K_{g}}{T_{g}{ }^{*} \omega_{0}} \omega_{H}+\frac{K_{g}}{T_{g}}, \\
\frac{d P_{H}}{d t} & =\frac{F_{H}}{T_{c h}} C_{V}-\frac{1}{T_{c h}} P_{H}, \\
\frac{d P_{I}}{d t} & =\frac{F_{I}}{T_{r h}{ }^{*} F_{H}} P_{H}-\frac{1}{T_{r h}} P_{I}, \\
\frac{d P_{A}}{d t} & =\frac{F_{A}}{T_{c 0}{ }^{*} F_{I}} P_{I}-\frac{1}{T_{c 0}} P_{A},
\end{aligned}
$$

where $P_{H}, P_{I}$, and $P_{A}$ are mechanical powers applied by the governor to different turbine parts, and $C_{V}$ is vapor valve in governor.

\subsubsection{Excitation system}

Block diagram for the excitation system is shown in Figure 7 [34]. Relationship between output voltage of the excitation system and field voltage is expressed as in $E_{f d}=\left(L_{a d} / R_{f d}\right) e_{f d}$.

According to the block diagram, equations of excitation system are written as follows:

$$
\begin{aligned}
& \frac{d e_{f d}}{d t}=-\frac{K_{E}}{T_{E}} e_{f d}+\frac{1}{T_{E}} \frac{R_{f d}}{L_{a d}} E_{R}, \\
& \frac{d E_{R}}{d t}=-\frac{1}{T_{A}} E_{R}-\frac{K_{A}}{T_{A}} E_{S B}-\frac{K_{A}}{T_{A}} V_{t}+\frac{K_{A}}{T_{A}} E_{\mathrm{ref}}, \\
& \frac{d E_{S B}}{d t}=-\frac{K_{E}{ }^{*} K_{F}{ }^{*} L_{a d}}{T_{E}{ }^{*} T_{F}{ }^{*} R_{f d}} e_{f d}+\frac{K_{F}}{T_{E}{ }^{*} T_{F}} E_{R}-\frac{1}{T_{F}} E_{S B},
\end{aligned}
$$

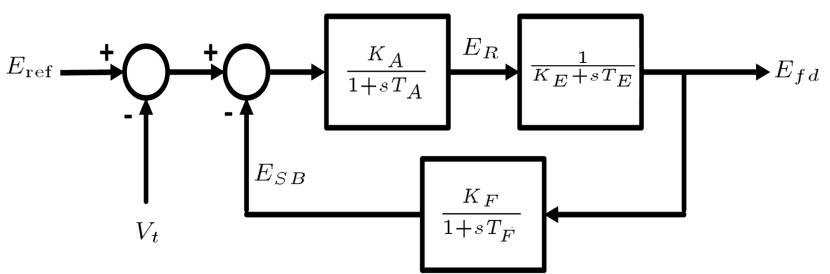

Figure 7. Block diagram of the excitation system. 


$$
\begin{aligned}
V_{t}= & \sqrt{V_{d}^{2}+V_{q}^{2}} \\
V_{d}= & V_{c d}+\frac{X_{T}}{\omega_{0}} \frac{d_{i d}}{d t}+R_{T}^{*} i_{d}-\frac{\omega}{\omega_{0}} X_{T}^{*} i_{q}+V_{b}^{*} \sin \delta \\
& +V_{\mathrm{inj} d} \\
V_{q}= & V_{c q}+\frac{X_{T}}{\omega_{0}} \frac{d_{i q}}{d t}+R_{T}^{*} i_{q}+\frac{\omega}{\omega_{0}} X_{T}^{*} i_{d}+V_{b}^{*} \sin \delta \\
& +V_{\mathrm{inj} q}, \\
R_{T}= & R_{t}+R_{L}+R_{s} \\
X_{T}= & X_{t}+X_{L}+X_{s}+X_{\mathrm{sys}} .
\end{aligned}
$$

The vector of state variables for mechanical side and excitation is as follows:

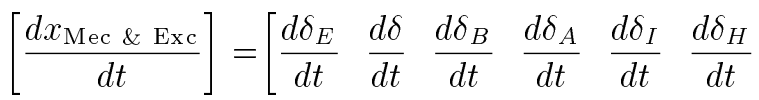

$$
\begin{aligned}
& \frac{d \omega_{E}}{d t} \quad \frac{d \omega}{d t} \quad \frac{d \omega_{B}}{d t} \quad \frac{d \omega_{A}}{d t} \quad \frac{d \omega_{I}}{d t} \quad \frac{d \omega_{H}}{d t} \\
& \frac{d C_{v}}{d t} \quad \frac{d P_{H}}{d t} \quad \frac{d P_{I}}{d t} \quad \frac{d P_{A}}{d t} \quad \frac{d e_{f d}}{d t} \\
& \left.\frac{d E_{R}}{d t} \frac{d E_{S B}}{d t}\right]^{T} .
\end{aligned}
$$

\subsection{SSSC-based stabilizers}

To control the magnitude and phase of the injected voltage, modulation ratio $(m)$ and angle $(\varphi)$ can be controlled. The block diagrams of these controllers are shown in Figures 8 and 9, respectively.

The equations derived from the above block diagrams can be written as follows:

$$
\begin{aligned}
& \frac{d m}{d t}=\frac{1}{T_{\mathrm{sssc}}}\left(m_{\mathrm{ref}}-m+U_{m}\right), \\
& \frac{d \varphi}{d t}=\frac{1}{T_{\mathrm{sssc}}}\left(\varphi_{\mathrm{ref}}-\varphi+U_{\varphi}\right),
\end{aligned}
$$

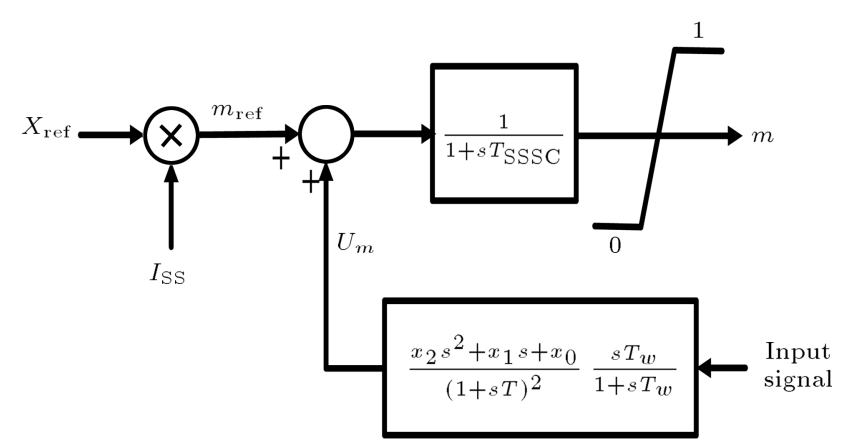

Figure 8. A SSSC magnitude controller with a damping stabilizer.

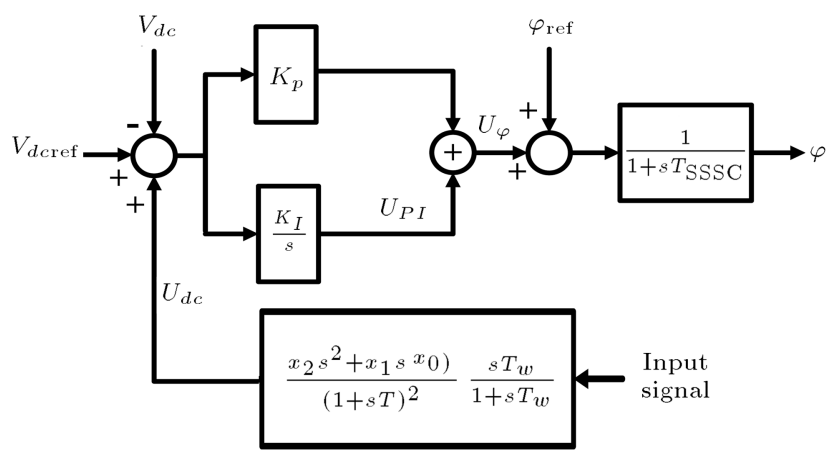

Figure 9. A SSSC phase controller with a damping stabilizer.

$$
\begin{aligned}
& \frac{d U_{P I}}{d t}=K_{I}\left(V_{d c \mathrm{ref}}-V_{d c}+U_{d c}\right) \\
& U_{\varphi}=U_{P I}+K_{P}\left(V_{d c \text { ref }}-V_{d c}+U_{d c}\right),
\end{aligned}
$$

where $\varphi_{\text {ref }}$ and $m_{\text {ref }}$ are the angle and magnitude of the injected voltage by the SSSC in the steady-state condition, respectively:

$$
m_{\mathrm{ref}}=X_{\mathrm{ref}}^{*} I_{\mathrm{ss}},
$$

where $X_{\text {ref }}$ is the reactance of SSSC and Iss is the current passing through SSSC in the steady-state conditions. When the magnitude-based stabilizer is used, $U_{d c}$ is set to zero; when the phase-based stabilizer is used, $U_{m}$ is set to zero. The vector of state variables for electrical, exciter, and mechanical sides of the system together with SSSC stabilizers is as follows:

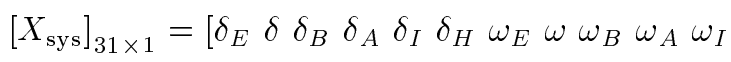

$$
\begin{aligned}
& \omega_{H} C_{v} P_{H} P_{I} P_{A} e_{f d} E_{R} E_{S B} i_{d} i_{q}
\end{aligned}
$$

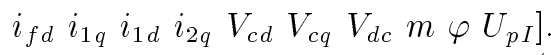

\section{Cause of SSR}

In order to analyze SSR in the steady-state conditions, the eigenvalues have been analyzed. Analysis of the eigenvalues provided useful information about stability of the system modes. Torsional modes depend on the intrinsic properties of generator's masses. The main cause of SSR phenomenon is excitation of torsional modes. This is caused by interaction and proximity between frequency of one of the torsional modes with that of sub-synchronous resonance mode. Due to the presence of series capacitor in the transmission lines, two electric modes are created in the system: subsynchronous and sup-synchronous modes. Torsional frequencies are always smaller than synchronous frequencies. Frequency of the system's electric mode $\left(f_{m}\right)$ and resonance frequency $\left(f_{s}\right)$ can be obtained as follows [2]: 


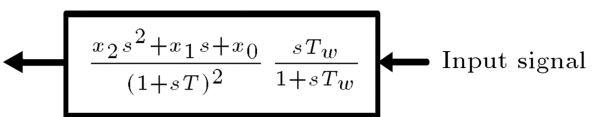

Figure 10. Block diagram of the stabilizer.

$$
\begin{aligned}
& f_{s}=f_{0} \sqrt{\frac{X_{C}}{X_{L}}}, \\
& f_{m}=f_{0} \pm f_{s},
\end{aligned}
$$

where $X_{L}$ is reactance of the transmission line, and $X_{C}$ is reactance of the series capacitor. $f_{0}$ is the synchronous frequency, $f_{m}=f_{0}+f_{s}$, is the system's super-synchronous frequency, while $f_{m}=f_{0}-f_{s}$ is its sub-synchronous frequency. In order to prevent SSR phenomenon, interaction of sub-synchronous electric mode with torsional modes must be prevented. In this paper, the main purpose of SSSC stabilizer is to keep the sub-synchronous mode away from the system's torsional mode.

\section{Stabilizer design}

Block diagram shown in Figure 10 was used for a SSSC stabilizer. In this paper, rotor speed is selected as an input signal for the stabilizer.

The following lead-lag stabilizer is considered for SSSC [28]:

$$
f(s)=\frac{x_{2} s^{2}+x_{1} s+x_{0}}{(1+s T)^{2}} \frac{s T_{w}}{1+s T_{w}} .
$$

The terms $x_{0}, x_{1}, x_{2}>0$ are adjustable parameters for stabilizer, $T$ is time constant, and $T_{w}$ is wash-out time. The values of $T$ and $T_{w}$ are chosen 0.3 and 10 , respectively.

\subsection{Objective function}

In this paper, the objective function used to control SSR is composed of four parts:

1. The first objective function: The first considered objective function is the magnitude of the stabilizer function, which can be expressed as follows:

$$
F_{1}=\sum_{i=1}^{N}\left|f\left(j \omega_{i}\right)\right|,
$$

where $\omega_{1}, \omega_{2}, \cdots, \omega_{N}$ are a set of frequencies in the region in which the critical mode should be shifted.

2. The second and third objective functions: The second and third objective functions are related to displacement of critical modes with the least damping coefficients and real parts close to imaginary axis. These functions are considered as Eqs. (55) and (56). A D-shape sector for these functions in the s-plane is shown in Figure 11 [36]:

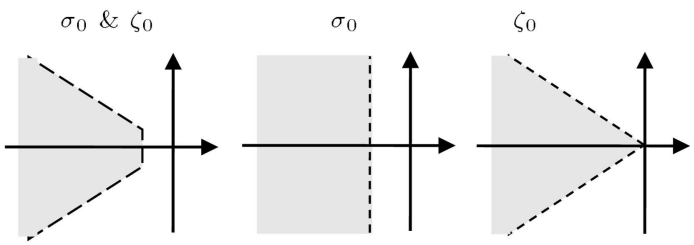

Figure 11. Range of displacement of the modes.

$$
\begin{aligned}
& F_{2}=\sum_{\sigma_{0} \leq \sigma_{1}}^{n}\left(\sigma_{0}-\sigma_{1}\right)^{2}, \\
& F_{3}=\sum_{\zeta_{0} \leq \zeta_{1}}^{n}\left(\zeta_{0}-\zeta_{1}\right)^{2}, \\
& \lambda_{i}=\sigma_{i} \pm \omega_{i} \\
& \zeta_{i}=\frac{-\sigma_{i}}{\sqrt{\sigma_{i}^{2}+\omega_{i}^{2}}},
\end{aligned}
$$

where $\sigma_{i}$ and $\zeta_{i}$ are the real parts and the damping coefficient of $i$ th critical mode, $\sigma_{0}$ and $\zeta_{0}$ are constant values to which the selected eigenvalues in the intended range should get closer to these values.

3. The fourth objective function: The constraints of the problem are related to parameters $x_{0}, x_{1}, x_{2}>$ 0 of the stabilizer. The constraints are related to stabilizer's zeroes. At first, the stabilizer's transfer function is considered as Eq. (59) by these changes of parameters: $\hat{x}_{1}=x_{1} / x_{0} T, \hat{x}_{2}=x_{2} / x_{0} T^{2}$ and $\bar{s}=s T:$

$$
F(\bar{s})=x_{0} \frac{\widehat{x}_{2} \bar{s}^{2}+\widehat{x}_{1} \bar{s}+1}{(1+\bar{s})^{2}} \frac{T_{w} \bar{s}}{1+T_{W} \bar{s}} .
$$

Transfer function, $F(\bar{s})$, has a pair of poles at $s=-1$. For the phase-lead structure, we assume that the zeros of $F(\bar{s})$ with respect to its poles are almost one-decade closer to the origin, meaning that they belong to the interval $[-0.1-1]$; for the phase-lag structure, zeros are almost one-decade away from the origin, meaning that they belong to interval $[-10-1]$. With some simplifications, the constraints for phase-lead structure can be written as follows:

$$
\begin{aligned}
& \widehat{x}_{1}-\widehat{x}_{2} \leq 1, \\
& \widehat{x}_{1}-0.1 \widehat{x}_{2} \leq 10, \\
& -99 \widehat{x}_{1}+18 \widehat{x}_{2} \leq-180 .
\end{aligned}
$$

By substituting $\hat{x}_{1}=x_{1} / x_{0} T$ and $\hat{x}_{2}=x_{2} / x_{0} T^{2}$ into Eqs. (60) to (62), the constraints for the lead state can be rewritten as follows:

$$
\begin{aligned}
& T x_{1}-x_{2}-x_{0} T^{2} \leq 0 \\
& T x_{1}-0.1 x_{2}-10 x_{0} T^{2} \leq 0,
\end{aligned}
$$




$$
-99 x_{1} T+18 x_{2}-180 x_{0} T^{2} \leq 0 .
$$

To apply the effect of these constraints on the objective function, the above equations are considered as follows:

$$
\begin{aligned}
& G_{1}(x)=T x_{1}-T^{2} x_{0}-x_{2}, \\
& G_{2}(x)=T x_{1}-10 T^{2} x_{0}-0.1 x_{2}, \\
& G_{3}(x)=4 x_{2} x_{0}-x_{1}^{2} .
\end{aligned}
$$

With a similar method, the equations for the stabilizer with phase-lag structure are considered as in:

$$
\begin{aligned}
& G_{1}(x)=T x_{1}-T^{2} x_{0}-x_{2}, \\
& G_{2}(x)=T x_{1}-0.1 T^{2} x_{0}-10 x_{2}, \\
& G_{3}(x)=4 x_{2} x_{0}-x_{1}^{2} .
\end{aligned}
$$

The above equations are added to the objective function as a penalty factor according to Eq. (72):

$$
F_{4}=\sum_{i=1}^{2} K_{i} G_{i}(x)^{2}
$$

where $K_{i}$ is a coefficient calculated with respect to the value of $G_{i}(x)$ according to Eq. (73):

$$
K_{i}= \begin{cases}50 & \text { if } G_{i}(x)>0 \\ 0 & \text { otherwise }\end{cases}
$$

The above functions and constraints are considered as in the following objective function:

$$
\text { M.O.F }=\sum_{i=1}^{4} w_{i} F_{i}=w_{1} F_{1}+w_{2} F_{2}+w_{3} F_{3}+w_{4} F_{4},
$$

where $w_{i}$ is constant weighting coefficients selected, empirically considering the impact on the objective function value. In this paper, these coefficients are considered to be $1,150,10$, and 50 , respectively. The following limits have been considered for variables of the SSSC-based stabilizer:

$$
\begin{aligned}
& x_{0, \min } \leq x_{0} \leq x_{0, \max }, \quad x_{1, \min } \leq x_{1} \leq x_{1, \max }, \\
& x_{2, \min } \leq x_{2} \leq x_{2, \max } .
\end{aligned}
$$

\section{GWO Algorithm}

Grey wolf optimization algorithm is a new populationbased algorithm introduced in 2014 by Mirjalili et al. [37,38]. Grey wolf (Canis lupus) belongs to Canidae family. Of particular interest is that they have a very strict social dominant hierarchy, as shown in Figure 12. The alpha is mostly responsible for making decisions

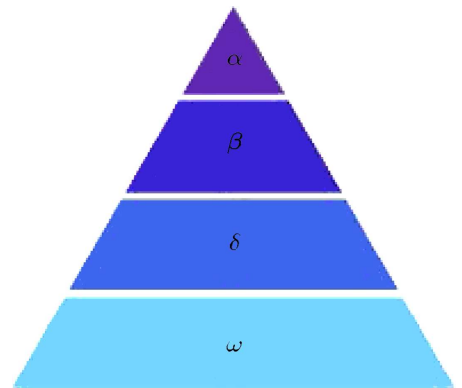

Figure 12. Hierarchy of grey wolf (dominance decreases from top down).

about hunting, sleeping place, time to wake, and so on. The second level in the hierarchy of grey wolves is beta. The betas are subordinate wolves that help the alpha in decision-making or other pack activities. The beta wolf is probably the best candidate to be the alpha in case one of the alpha wolves passes away or becomes very old. Omega is the lowest ranking grey wolf. The omega wolves play the role of scapegoat. Omega wolves always have to submit to all the other dominant wolves. They are the last wolves that are allowed to eat. If a wolf is not an alpha, beta, or omega, he/she is called subordinate (or delta in some references). Delta wolves have to submit to alphas and betas, but they dominate the omega. Scouts, sentinels, elders, hunters, and caretakers belong to this category.

The main phases of grey wolf hunting are as follows:

- Tracking, chasing, and approaching the prey;

- Pursuing, encircling, and harassing the prey until it stops moving;

- Attacking the prey.

In order to model the social hierarchy of wolves mathematically when designing GWO, we should consider the fittest solution as alpha $(\alpha)$. Consequently, the second and third best solutions are named beta $(\beta)$ and delta $(\delta)$, respectively. The rest of the candidate solutions are assumed to be omega $(\omega)$.

Grey wolves encircle the prey during the hunt. In order to model the encircling behavior mathematically, the following equations are proposed:

$$
\begin{aligned}
& \vec{D}=\left|\vec{C} \cdot \vec{X}_{P}(t)-\vec{X}(t)\right|, \\
& \vec{X}(t+1)=\vec{X}_{P}(t)-\vec{A} \vec{D},
\end{aligned}
$$

where $t$ indicates the current iteration, $\vec{A}$ and $\vec{C}$ are coefficient vectors, $\vec{X}_{P}$ is the position vector of the prey, and $\vec{X}$ indicates the position vector of a grey wolf.

Vectors $\vec{A}$ and $\vec{C}$ are calculated as follows:

$$
\begin{aligned}
& \vec{A}=2 \vec{a} \vec{r}_{1}-\vec{a}, \\
& \vec{C}=2 \vec{r}_{2},
\end{aligned}
$$


where components of $\vec{a}$ are linearly decreased from 2 to 0 over the course of iterations, and $r_{1}, r_{2}$ are random vectors in $[0,1]$. Grey wolves have the ability to recognize the location of prey and encircle it. In order to simulate the hunting behavior of grey wolves mathematically, we suppose that the alpha (the best candidate solution), beta, and delta have better knowledge about the potential location of the prey. Therefore, we save the first three best solutions obtained so far and oblige the other search agents (including the omegas) to update their positions according to the position of the best search agents. The following formulas are proposed in this regard:

$$
\begin{aligned}
& \vec{D}_{\alpha}=\left|\vec{C}_{1} \vec{X}_{\alpha}-\vec{X}\right|, \quad \vec{D}_{\beta}=\left|\vec{C}_{2} \vec{X}_{\beta}-\vec{X}\right|, \\
& \vec{D}_{\delta}=\left|\vec{C}_{3} \vec{X}_{\delta}-\vec{X}\right|, \\
& \vec{X}_{1}=\vec{X}_{\alpha}-\vec{a}_{1} \vec{D}_{\alpha}, \quad \vec{X}_{2}=\vec{X}_{\beta}-\vec{a}_{2} \vec{D}_{\beta}, \\
& \vec{X}_{3}=\vec{X}_{\delta}-\vec{a}_{3} \vec{D}_{\delta}, \\
& \vec{X}(t+1)=\frac{\vec{X}_{1}+\vec{X}_{2}+\vec{X}_{3}}{3} .
\end{aligned}
$$

The final position would be in a random position within a circle which is defined by the positions of alpha, beta, and delta in the search space. In other words, alpha, beta, and delta estimate the victim position and other wolves update their positions randomly around the victim [39].

Different steps in applying GWO algorithm to minimize the proposed objective function are as follows:

Step 1. Define input data of the studied power system and necessary parameters of the SSSC-based stabilizer;

Step 2. Determine the number of search agents $(N)$ and maximum number of iterations (iter-max);

Step 3. Initialise the gray wolf population matrix $(X)$. In this matrix, each population set represents the position of a search agent. From the optimization point of view, position of a search agent signifies one of candidates for the minimization of the objective function. In the proposed objective function, position of each search agent consists of three unspecific parameters of the SSSC stabilizer (i.e., $x_{2}, x_{1}$, and $x_{0}$ as shown in Figure 10). Each element of the position of search agent is initialized within the limits of parameters of the SSSC stabilizer and may be determined as follows:

$$
x_{m, j}=x_{m, j}^{\min }+\operatorname{rand}(0,1) \times\left(\max _{m, j}-x_{m, j}^{\min }\right),
$$

where $x_{m, j}$ is $j$ th element of $m$ th search agent position. Here, $m=1,2, \cdots, N$ and $j=1,2, \cdots, D$. Here, $N$ is the maximum number of the search agents and $D$ is the number of variable in the problem (in the proposed objective function $D=3$ ). According to the above explanations, matrix $X$ can be presented in the following way:

$$
X=\left[\begin{array}{c}
X_{1} \\
X_{2} \\
\vdots \\
X_{N}
\end{array}\right]=\left[\begin{array}{ccc}
x_{1,1} & x_{1,2} & x_{1,3} \\
x_{2,1} & x_{2,2} & x_{2,3} \\
\vdots & \vdots & \vdots \\
x_{N, 1} & x_{N, 2} & x_{N, 3}
\end{array}\right]
$$

Position of each search agent should satisfy the constraints of parameters of SSSC stabilizer;

Step 4. If constraints limits of each search agent of matrix $X$ are satisfied, then go to the next step, otherwise go back to Step 3;

Step 5. Initialise a, A, and C using Eqs. (78) and (79);

Step 6. Linearise the power system installed with a SSSC-based stabilizer for each search agent, calculate the aginvalues and determine the critical modes, and then evaluate the fitness function value of each search agent;

Step 7. Set the positions of search agent of matrix $X$ corresponding to the first, second, and third values of fitness function as $X_{\alpha}, X_{\beta}$, and $X_{\delta}$, respectively;

Step 8. Set iteration number iter $=1$;

Step 9. Update the position of each search agent using Eqs. (80)-(82);

Step 10. Update the values of a, A, and C using Eqs. (78) and (79);

Step 11. Check the limits of parameters of the SSSC-based stabilizer;

Step 12. If constraints limits are satisfied, then go to the next step, otherwise go to Step 9 again and repeat Steps 9-12;

Step 13. Linearise power system installed with SSSC-based stabilizer for each updated search agent, calculate the aigenvalues and determine the critical modes, and then evaluate the fitness function value of each updated search agent;

Step 14. Set the position of search agent corresponding to the first, second, and third values of fitness function as $X_{\alpha}, X_{\beta}$, and $X_{\delta}$, respectively;

Step 15. Increase iteration number by 1 , that is, iter $=$ iter +1 ;

Step 16. If the maximum number of iteration is reached, stop the iterative process and store $X_{\alpha}$ as the best solution to the optimization problem; otherwise, repeat Steps 9-16. 

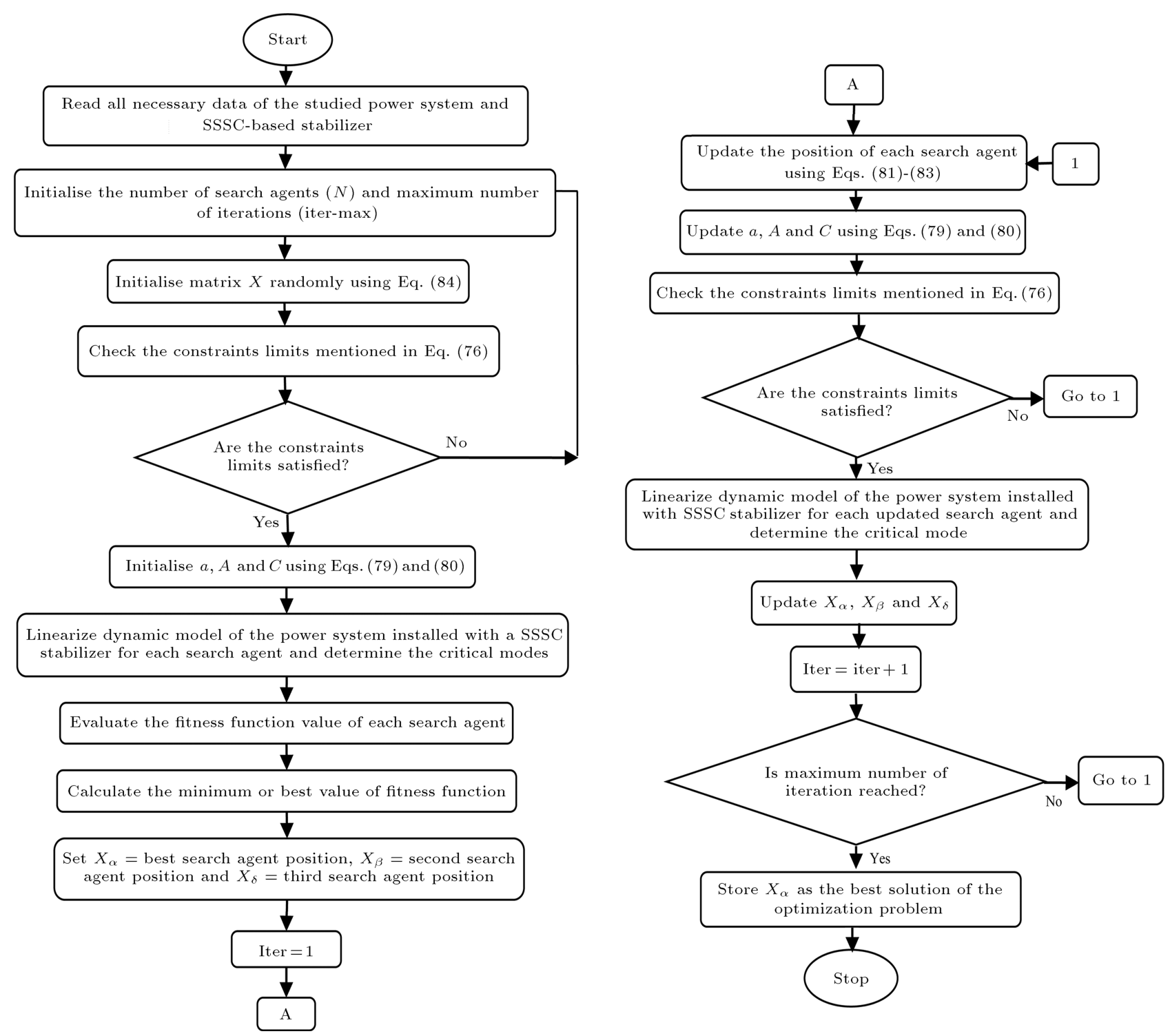

Figure 13. Flowchart of the GWO algorithm.

Flowchart of the algorithm is shown in its simplest form in Figure 13.

\section{Simulation results}

\subsection{Analysis of eigenvalues}

The studied system has been linearized about the values of operation conditions. The needed data for simulations of the system are presented in the Appendix. Data of mechanical system are given in Table A.1. The eigenvalues of the open-loop system without a SSSC and with a SSSC for different compensation degrees are shown in Table 1. This table shows that when the frequency of the torsional mode is close to the that of the sub-synchronous mode, the torsional mode will be unstable and SSR phenomenon occurs. With the increase of compensation degrees, resonance frequency $\left(f_{s}\right)$ increases and the frequency of the sub-synchronous mode decreases. Since the torsional modes depend on the intrinsic properties of generator's masses, the frequency of the torsional mode is nearly constant. With the presence of a SSSC (without a stabilizer) in capacitive mode in steadystate conditions, the total capacitive reactance of the transmission line is increased. Therefore, the resonance frequency is increased, and consequently, the subsynchronous frequency is decreased. In the case with a SSSC, the SSR phenomenon occurs, too.

To prevent SSR phenomenon, a lead-lag stabilizer is included into the SSSC in different control channels. The parameters of the stabilizer for different compensation degrees in the phase-based stabilizer and the magnitude-based stabilizer are shown in Tables 2 and 3 , respectively. In these tables, the eigenvalues of the critical torsional mode and its damping coefficient are shown. The values show that the system is stable 
Table 1. Eigenvalues of the open-loop system with and without a SSSC.

\begin{tabular}{|c|c|c|c|c|c|c|}
\hline \multirow[b]{2}{*}{ Mode } & \multicolumn{2}{|c|}{$\begin{array}{c}\text { Compensation } \\
\text { degree }=41.1 \%\end{array}$} & \multicolumn{2}{|c|}{$\begin{array}{c}\text { Compensation } \\
\text { degree }=54.7 \%\end{array}$} & \multicolumn{2}{|c|}{$\begin{array}{c}\text { Compensation } \\
\text { degree }=68.5 \%\end{array}$} \\
\hline & $\begin{array}{l}\text { Without } \\
\text { SSSC }\end{array}$ & $\begin{array}{c}\text { SSSC } \\
\text { without } \\
\text { stabilizer }\end{array}$ & $\begin{array}{l}\text { Without } \\
\text { SSSC }\end{array}$ & $\begin{array}{c}\text { SSSC } \\
\text { without } \\
\text { stabilizer }\end{array}$ & $\begin{array}{l}\text { Without } \\
\text { SSSC }\end{array}$ & $\begin{array}{c}\text { SSSC } \\
\text { without } \\
\text { stabilizer }\end{array}$ \\
\hline 5 & $-0.5 \pm 298.28 i$ & $-0.5 \pm 298.28 i$ & $-0.5 \pm 298.28 i$ & $-0.5 \pm 298.28 i$ & $-0.49 \pm 298.28 i$ & $-0.5 \pm 298.28 i$ \\
\hline 4 & $-0.10 \pm 202.83 i$ & $-0.12 \pm 202.74 i$ & $-0.11 \pm 202.90 i$ & $-0.12 \pm 202.85 i$ & $-0.11 \pm 202.94 i$ & $-0.12 \pm 202.89 i$ \\
\hline 3 & $0.95 \pm 160.67 i$ & $0.27 \pm 161.96 i$ & $-0.43 \pm 160.50 i$ & $-0.52 \pm 160.43 i$ & $-0.44 \pm 160.58 i$ & $-0.52 \pm 160.54 i$ \\
\hline 2 & $-0.14 \pm 127.09 i$ & $-0.15 \pm 127.09 i$ & $0.59 \pm 127.13 i$ & $0.06 \pm 127.58 i$ & $-0.14 \pm 126.99 i$ & $-0.15 \pm 126.97 i$ \\
\hline 1 & $-0.21 \pm 99.38 i$ & $-0.25 \pm 99.38 i$ & $-0.18 \pm 99.88 i$ & $-0.23 \pm 99.78 i$ & $4.50 \pm 98.97 i$ & $4.41 \pm 100.59 i$ \\
\hline $\mathbf{0}$ & $-0.50 \pm 9.26 i$ & $-0.43 \pm 9.02 i$ & $-0.59 \pm 10.28 i$ & $-0.5 \pm 9.58 i$ & $-0.73 \pm 11.62 i$ & $-0.63 \pm 10.69 i$ \\
\hline $\begin{array}{c}\text { Sub- } \\
\text { synchronous }\end{array}$ & $-3.90 \pm 160.8 i$ & $-2.78 \pm 163.43 i$ & $-2.50 \pm 127.29 i$ & $-1.39 \pm 130.23 i$ & $-5.38 \pm 98.94 i$ & $-5.18 \pm 100.68 i$ \\
\hline $\begin{array}{c}\text { Super- } \\
\text { synchronous }\end{array}$ & $-4.81 \pm 593.08 i$ & $-3.06 \pm 589.19 i$ & $-4.86 \pm 626.30 i$ & $-3.09 \pm 622.95 i$ & $-4.9 \pm 655.78 i$ & $-3.11 \pm 652.23 i$ \\
\hline
\end{tabular}

Table 2. Parameters of the proposed phase-based stabilizer.

\begin{tabular}{ccccccc}
\hline Compensation degree & $\boldsymbol{x}_{\mathbf{0}}$ & $\boldsymbol{x}_{\mathbf{1}}$ & $\boldsymbol{x}_{\boldsymbol{2}}$ & Critical mode & $\boldsymbol{\%} \boldsymbol{\zeta}$ & $\left|\boldsymbol{f}_{\boldsymbol{\varphi}}\left(\boldsymbol{j}{\overline{\omega_{p}}}\right)\right|$ \\
\hline $\mathbf{4 1 . 1 \%}$ & 8.0010 & 2.0542 & 0.0004 & $-0.50 \pm 161.04 i$ & 0.3127718 & 0.1426 \\
$\mathbf{5 4 . 7 \%}$ & 8.0012 & 1.9214 & 0.0003 & $-0.14 \pm 127.17 i$ & 0.1149188 & 0.1680 \\
$\mathbf{6 8 . 5 \%}$ & 8.0029 & 1.3865 & 0.0001 & $-0.11 \pm 100.70 i$ & 0.1176916 & 0.1556 \\
\hline
\end{tabular}

Table 3. Parameters of the proposed magnitude-based stabilizer.

\begin{tabular}{ccccccc}
\hline Compensation degree & $\boldsymbol{x}_{\mathbf{0}}$ & $\boldsymbol{x}_{\mathbf{1}}$ & $\boldsymbol{x}_{\mathbf{2}}$ & Critical mode & $\boldsymbol{\%} \boldsymbol{\zeta}$ & $\left|\boldsymbol{f}_{\boldsymbol{m}}\left(\boldsymbol{j} \overline{\boldsymbol{\omega}_{\boldsymbol{p}}}\right)\right|$ \\
\hline $\mathbf{4 1 . 1 \%}$ & 8.003 & 0.0002 & 0.0132 & $-0.52 \pm 161.08 i$ & 0.3586333 & 0.1431 \\
$\mathbf{5 4 . 7 \%}$ & 8.001 & 0.0001 & 0.0128 & $-0.15 \pm 127.17 i$ & 0.1195701 & 0.1366 \\
$\mathbf{6 8 . 5 \%}$ & 8.0016 & 0.0001 & 0.0103 & $-0.24 \pm 100.73 i$ & 0.2444158 & 0.1053 \\
\hline
\end{tabular}

and the damping of the critical torsional mode is acceptable.

In the last columns of Tables 2 and 3 , the values of the stabilizer in the frequency of the critical mode are shown. Comparing the values, for the SSSC stabilizer in two control channels in different compensation degrees, shows that the stabilizer in the magnitude control channel is a little more effective in damp critical mode than the phase control channel. The SSSC stabilizer in the magnitude control channel with lower gain (a little) has better effect on the damping of critical torsional mode than the phase control channel. The eigenvalues of important modes in the closed-loop system are shown in Table 4 . This table shows that the critical torsional mode is stable and damping of other oscillation modes is acceptable.

\subsection{Non-linear simulations}

To certify the eigenvalues analysis, a fault is applied to the non-linear system. The fault is considered as a $10 \%$ step decrease in the input mechanical torque

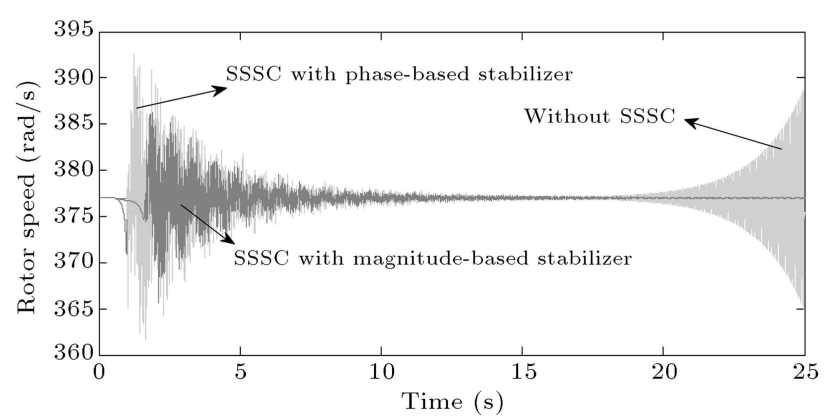

Figure 14. Variations of rotor speed for compensation degree $=54.7 \%$.

of LPA low-pressure turbine. The fault is started at $t=0.5 \mathrm{~s}$ and removed at $t=1 \mathrm{~s}$ (without any switching in the system). Variations of rotor speed, typically, for compensation degrees equal to $54.7 \%$ and $41.1 \%$ are shown in Figures 14 and 15, respectively. These figures show that the SSR phenomenon is created without a SSSC stabilizer, and amplitude of the oscillations is growing with the time and the system will be unstable, 
Table 4. Eigenvalues of the closed-loop system.

\begin{tabular}{|c|c|c|c|c|c|c|}
\hline \multirow[b]{2}{*}{ Mode } & \multicolumn{2}{|c|}{$\begin{array}{c}\text { Compensation } \\
\text { degree }=41.1 \%\end{array}$} & \multicolumn{2}{|c|}{$\begin{array}{c}\text { Compensation } \\
\text { degree }=54.7 \%\end{array}$} & \multicolumn{2}{|c|}{$\begin{array}{c}\text { Compensation } \\
\text { degree }=68.5 \%\end{array}$} \\
\hline & $\begin{array}{c}\varphi \text {-based } \\
\text { SSSC } \\
\text { stabilizer }\end{array}$ & $\begin{array}{c}m \text {-based } \\
\text { SSSC } \\
\text { stabilizer }\end{array}$ & $\begin{array}{c}\varphi \text {-based } \\
\text { SSSC } \\
\text { stabilizer }\end{array}$ & $\begin{array}{c}m \text {-based } \\
\text { SSSC } \\
\text { stabilizer }\end{array}$ & $\begin{array}{c}\varphi \text {-based } \\
\text { SSSC } \\
\text { stabilizer }\end{array}$ & $\begin{array}{c}m \text {-based } \\
\text { SSSC } \\
\text { stabilizer }\end{array}$ \\
\hline 5 & $-0.5 \pm 298.28 i$ & $-0.5 \pm 298.28 i$ & $-0.5 \pm 298.28 i$ & $-0.5 \pm 298.28 i$ & $-0.5 \pm 298.28 i$ & $-0.5 \pm 298.28 i$ \\
\hline 4 & $-0.11 \pm 202.68 i$ & $-0.1 \pm 202.68 i$ & $-0.11 \pm 202.83 i$ & $-0.11 \pm 202.82 i$ & $-0.14 \pm 202.85 i$ & $-0.13 \pm 202.86 i$ \\
\hline 3 & $-0.5 \pm 161.04 i$ & $-0.52 \pm 161.08 i$ & $-0.43 \pm 160.16 i$ & $-0.41 \pm 160.16 i$ & $-0.6 \pm 160.45 i$ & $-0.54 \pm 160.48 i$ \\
\hline 2 & $-0.14 \pm 127.06 i$ & $-0.14 \pm 127.06 i$ & $-0.14 \pm 127.17 i$ & $-0.15 \pm 127.17 i$ & $-0.1 \pm 126.64 i$ & $-0.1 \pm 126.74 i$ \\
\hline 1 & $-0.18 \pm 99.06 i$ & $-0.21 \pm 99.07 i$ & $-0.16 \pm 99.19 i$ & $-0.2 \pm 99.21 i$ & $-0.11 \pm 100.70 i$ & $-0.24 \pm 100.73 i$ \\
\hline $\mathbf{0}$ & $-0.71 \pm 7.23 i$ & $-0.47 \pm 6.75 i$ & $-0.76 \pm 8.13 i$ & $-0.53 \pm 7.42 i$ & $-0.45 \pm 8.37 i$ & $-0.48 \pm 8.14 i$ \\
\hline $\begin{array}{c}\text { Sub- } \\
\text { synchronous }\end{array}$ & $-1.86 \pm 181.56 i$ & $-1.84 \pm 181.55 i$ & $-1.52 \pm 151.91 i$ & $-1.53 \pm 151.91 i$ & $-1.09 \pm 119.95 i$ & $-1.04 \pm 120.2 i$ \\
\hline $\begin{array}{c}\text { Super- } \\
\text { synchronous }\end{array}$ & $-3.04 \pm 572.54 i$ & $-3.05 \pm 574.7 i$ & $-3.07 \pm 602.58 i$ & $-3.07 \pm 602.58 i$ & $-3.1 \pm 633.35 i$ & $-3.1 \pm 633.33 i$ \\
\hline
\end{tabular}

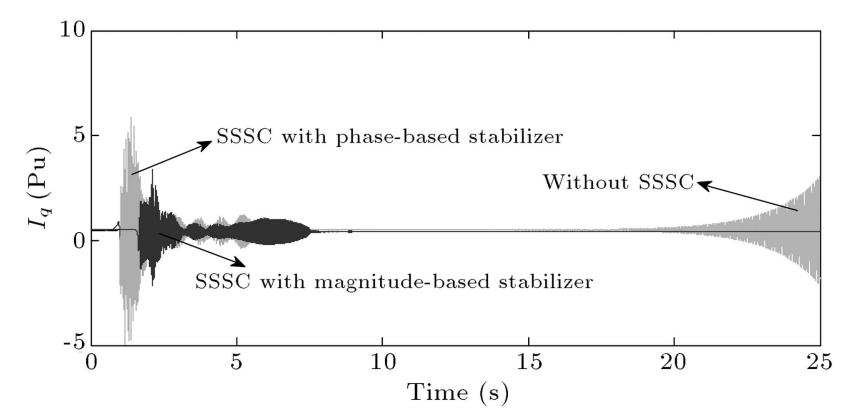

Figure 15. Variations of $I_{q}$ current for compensation degree $=54.7 \%$.

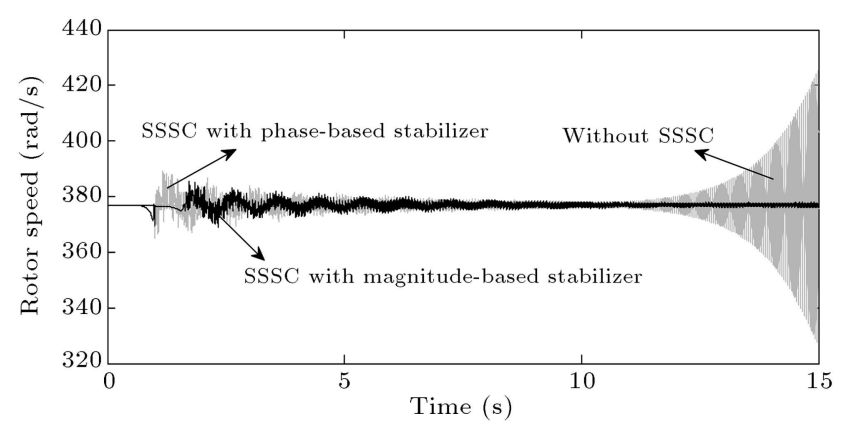

Figure 16. Variations of rotor speed for compensation degree $=41.1 \%$.

lastly. However, with the presence of a SSSC stabilizer in both control channels, the SSR phenomenon does not occur. This result is verified for variations of $I_{q}$ current in Figures 16 and 17.

Variations of the DC link voltage of SSSC in the phase control channel and the magnitude control channel are shown in Figures 18 and 19, respectively. These figures show that voltage of the DC link of the SSSC is returned to its steady-state value after a short time.

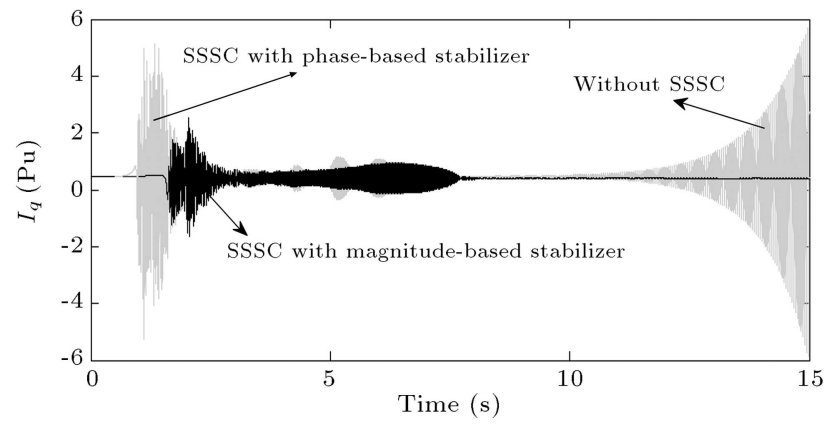

Figure 17. Variations of $I_{q}$ current for compensation degree $=41.1 \%$

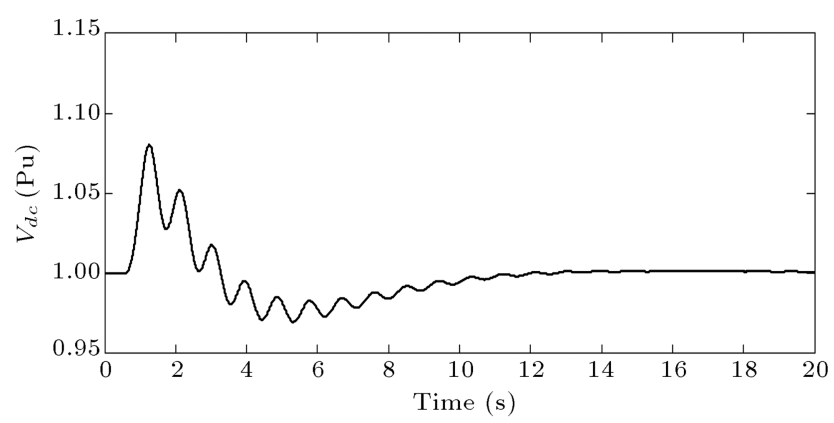

Figure 18. Variations of the DC link voltage with a phase-based stabilizer for compensation degree $=54.7 \%$.

\section{Conclusion}

In this article, SSR phenomenon in a single machine power system with the presence of a SSSC was investigated. The studied system was modeled in $d-q$ from. To design a SSSC-based stabilizer, a new multi-objective function was proposed. In this function, shifting of critical modes to suitable area and also the gain of stabilizer were considered. By this method, in addition to improving the stability of 


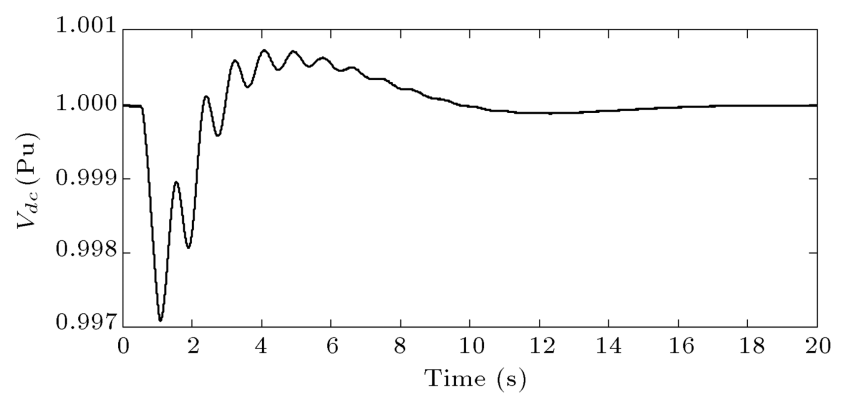

Figure 19. Variations of the DC link voltage with a magnitude-based stabilizer for compensation degree $=$ $54.7 \%$.

SSR oscillations, the control cost of the stabilizer was reduced. To have a stabilizer with minimum phase structure, appropriate constraints were determined and considered in the function. To design the parameters of the stabilizer, GWO algorithm was used. The effects of the SSSC stabilizer in different control channels on SSR oscillations were studied. The eigenvalues analysis and nonlinear simulation results showed that the SSSC stabilizer in both control channels could effectively improve the damping of SSR oscillations. The results were confirmed at different levels of series compensation. By comparing the results, it can be concluded that the SSSC stabilizer in magnitude control channel is a little more effective in damping SSR oscillations than that in the phase control channel.

\section{References}

1. Mathur, R.M. and Varma, R.K., Thyristor-Based FACTS Controllers for Electrical Transmission Systems, John Wiley \& Sons (2002).

2. Padiyar, K., Analysis of Subsynchronous Resonance in Power Systems, 471, Springer (1998).

3. Virulkar, V.B. and Gotmare, G.V. "Sub-synchronous resonance in series compensated wind farm: A review", Renewable and Sustainable Energy Reviews, 55, pp. 1010-1029 (2016).

4. Widyan, M.S. "On the effect of AVR gain on bifurcations of subsynchronous resonance in power systems", Int. J. Elect. Power and Energy Syst., 32(6), pp. 656663 (2010).

5. Ghorbani, A. and Pourmohammad, S. "A novel excitation controller to damp subsynchronous oscillations", Int. J. Elect. Power \& Energy Syst., 33(3), pp. 411-419 (2011).

6. De Jesus, F.D., Watanabe, E.H., and De Souza, L.F.W. "SSR and power oscillation damping using gate-controlled series capacitors (GCSC)", IEEE Trans. Power Del., 22(3), pp. 1806-1812 (2007).

7. Mokhtari, M., Khazaei, J. and Nazarpour, D. "Subsynchronous resonance damping via doubly fed induction generator", Int. J. Elect. Power and Energy Syst., 53, pp. 876-883 (2013).
8. Khazaie, J., Mokhtari, M., Khalilyan, M., et al. "Subsynchronous resonance damping using distributed static series compensator (DSSC) enhanced with fuzzy logic controller", Int. J. Elect. Power and Energy Syst., 43(1), pp. 80-89 (2012).

9. Zhao, B., Li, H., Wang, M., et al. "An active power control strategy for a DFIG-based wind farm to depress the subsynchronous resonance of a power system", Int. J. Elect. Power and Energy Syst., 69, pp. 327-334 (2015).

10. Mancilla-David, F., Dominguez-Garcia, J.L., De Prada, M., et al. "Modeling and control of Type-2 wind turbines for sub-synchronous resonance damping", Energy Conv. and Manag., 97, pp. 315-322 (2015).

11. Revela, G., Leon, A.E., Alonso, D.M., et al. "Multiparameter bifurcation analysis of subsynchronous interactions in DFIG-based wind farms", Electric Power Systems Research (in press).

12. Huang, P., Moursi, M., and Xiao, W. "Subsynchronous resonance mitigation for series-compensated DFIGbased wind farm by using two-degree-of-freedom control strategy", IEEE Trans. PWRS, 31(2), pp. 10381047 (2016).

13. Khayyatzadeh, M. and Kazemzadeh, R. "Subsynchronous resonance damping using high penetration PV plant", Mech. Syst. and Signal Proc., 84, pp. 431-444 (2017).

14. Wang, S., Xu, Z., and Wang, S. "New findings on bypass damping filter in increasing subsynchronous resonance damping of series compensated system", IET Gener. Transm. Distrib., 9(13), pp. 1718-1726 (2015).

15. Liu, H., Xie, X., and Wang, L. "Optimal design of linear subsynchronous damping controllers for stabilising torsional interactions under all possible operating conditions", IET Gener. Transm. Distrib., 9(13), pp. 1652-1662 (2015).

16. Adees, A. and Milanovic, J.V. "Methodology for evaluation of risk of subsynchronous resonance in meshed compensated networks", IEEE Trans. PWRS, 29(2), pp. 815-823 (2014).

17. Sen, K.K. "SSSC-static synchronous series compensator: theory, modeling, and application", IEEE Trans. Power Del., 13(1), pp. 241-246 (1998).

18. Bongiorno, M., Svensson, J., and Angquist, L. "On control of static synchronous series compensator for SSR mitigation", IEEE Trans. Power Electronics, 23(2), pp. 735-743 (2008).

19. Varma, R.K., Auddy, S., and Semsedini, Y. "Mitigation of subsynchronous resonance in a seriescompensated wind farm using FACTS controllers", IEEE Trans. Power Del., 23(3), pp. 1645-1654 (2008).

20. Khaparde, S. and Krishna, V. "Simulation of unified static VAr compensator and power system stabilizer for arresting subsynchronous resonance", In Power Industry Computer Applications, 20th International Conference on. IEEE (1997). 
21. El-Moursi, M.S., Bak-Jensen, B., and Abdel-Rahman, M.H. "Novel STATCOM controller for mitigating SSR and damping power system oscillations in a series compensated wind park", IEEE Trans. Power Electronics, 25(2), pp. 429-441 (2010).

22. Alomari, M.M. and Zhu, J.G. "Bifurcation control of subsynchronous resonance using TCSC", Communications in Nonlinear Science and Numerical Simulation, 16(5), pp. 2363-2370 (2011).

23. Thampatty, K.S., Nandakumar, M., and Cheriyan, E.P. "Adaptive RTRL based neurocontroller for damping subsynchronous oscillations using TCSC", Engineering Applications of Artificial Intelligence, 24(1), pp. 60-76 (2011).

24. Padiyar, K. and Prabhu, N. "Design and performance evaluation of subsynchronous damping controller with STATCOM", IEEE Trans. Power Del., 21(3), pp. 1398-1405 (2006).

25. Panda, S., Baliarsingh, A.K., Mahapatra, S. et al. "Supplementary damping controller design for SSSC to mitigate sub-synchronous resonance", Mech. Syst. and signal Prroc., 68-69, pp. 523-535 (2016).

26. Adees, A. and Milanovic, J.V. "Optimal compensation of transmission lines based on minimisation of the risk of subsynchronous resonance", IEEE Trans. PWERS, 30(3), pp. 1442-1454 (2015).

27. Rai, D., Faried, S.O., Ramakrishna, G., et al. "Hybrid series compensation scheme capable of damping subsynchronous resonance", IET Gener. Transm. Distrib., 4(3), pp. 456-466 (2010).

28. Shakarami, M. and Kazemi, A. "Simultaneous coordinated tuning of SSSC-based stabilizer and PSS using quadratic mathematical programming", Scientia Iranica, Trans. D, Comp. Science and Elec. Eng., 17(2), pp. 163-174 (2010).

29. Thirumalaivasan, R., Janaki, M., and Prabhu, N. "Damping of SSR using subsynchronous current suppressor with SSSC", IEEE Trans. PWRS, 28(1), pp. 64-74 (2013).

30. Rai, D., Ramakrishna, G., Faried, S.O. et al. "Enhancement of power system dynamics using a phase imbalanced series compensation scheme", IEEE Trans. PWRS, 25(2), pp. 966-974 (2010).

31. Farahani, M., Ganjefa, S., and Alizadeh, M. "Intelligent control of SSSC via an online self-tuning PID to damp the subsynchronous oscillations in Electrical Engineering (ICEE)", 2012 20th Iranian Conference on. IEEE (2012).

32. Group, I.S.W. "First benchmark model for computer simulation of subsynchronous resonance", IEEE Trans. on PAS, 96(5), pp. 1565-1572 (1977).

33. Padiyar, K. and Prabhu, N. "Analysis of SSR with three-level twelve-pulse VSC-based interline powerflow controller", IEEE Trans. Power Del., 22(3), pp. 1688-1695 (2007).

34. Kundur, P., Balu, N.J., and Lauby, M.G., Power System Stability and Control, 7, McGraw-Hill New York (1994).
35. Report, I. "Dynamic models for steam and hydro turbines in power system studies", IEEE Trans. PAS, 6, pp. 1904-1915 (1973).

36. Abdel-Magid, Y. and Abido, M. "Optimal multiobjective design of robust power system stabilizers using genetic algorithms", IEEE Trans. PWRS, 18(3), pp. 1125-1132 (2003).

37. Mirjalili, S., Mirjalili, S.M., and Lewis, A. "Grey wolf optimizer", Advances in Engineering Software, 69, pp. 46-61 (2014).

38. Sharma, S., Bhattacharjee, S., and Bhattacharya A. "Grey wolf optimisation for optimal sizing of battery energy storage device to minimize operation cost of microgrid", IET Gener. Transm. Distrib., 10(3), pp. 625-637 (2016).

39. Madadi, A. and Motlagh, M.M., Optimal Control of DC Motor Using Grey Wolf Optimizer Algorithm (2014).

\section{Appendix}

\section{Power system data:}

Generator data (in per unit on 892.4 MVA and $26 \mathrm{KV}$ base):

$$
\begin{aligned}
& R_{a}=1 e-7, \quad R_{f d}=0.0013, \quad R_{1 d}=0.0297 \\
& R_{1 q}=0.0124, \quad R_{2 q}=0.0182 \\
& X_{a d}=1.66, \quad X_{a q}=1.58 \\
& X_{d}=1.79, \quad X_{q}=1.71 \\
& X_{f f d}=1.7335, \quad X_{11 d}=1.7177 \\
& X_{11 q}=1.6319, \quad X_{22 q}=1.9029 .
\end{aligned}
$$

\section{Governor and turbine data:}

$$
\begin{aligned}
& K_{g}=25, \quad T_{g}=0.1 \mathrm{sec} \\
& T_{c h}=0.40 \mathrm{sec}, \quad T_{r h}=7.0 \mathrm{sec}, \quad T_{c o}=0.60 \mathrm{sec} \\
& F_{A}=0.22, \quad F_{B}=0.22 \\
& F_{I}=0.26, \quad F_{H}=0.30 \\
& C_{v \text { open }}=4.0 \mathrm{p} . \mathrm{u} . / \mathrm{sec}, \quad C_{v \text { close }}=4.0 \mathrm{p.u} . / \mathrm{sec} .
\end{aligned}
$$

\section{Excitation system data:}

$$
\begin{aligned}
& K_{A}=2, \quad K_{E}=1.0, \quad K_{F}=0.03 \\
& T_{A}=0.04 \mathrm{sec}, \quad T_{E}=0.01 \mathrm{sec}, \quad T_{F}=1.0 \mathrm{sec}, \\
& V_{R \max }=4.75 \mathrm{p} . \mathrm{u}, \quad V_{R \min }=-4.75 \mathrm{p} . \mathrm{u} .
\end{aligned}
$$


Table A.1. Mechanical system data.

\begin{tabular}{ccccc}
\hline Mass & Shaft & $\begin{array}{c}\text { Inertia, } \boldsymbol{M} \\
\text { (seconds) }\end{array}$ & $\begin{array}{c}\text { Damping, } \boldsymbol{D} \\
(\mathbf{p . u} . \mathbf{p . u . s p e e d})\end{array}$ & $\begin{array}{c}\text { Spring constant, } \boldsymbol{K} \\
(\mathbf{p . u} / \mathbf{r a d})\end{array}$ \\
\hline EXC & & 0.0684 & 0.017 & 2.822 \\
GEN & GEN-EXC & 1.736 & 0.099 & 70.858 \\
& LPB-GEN & 1.768 & 0.100 & 52.038 \\
LPB & & & & 34.929 \\
LPA & LPA-LPB & 1.716 & 0.100 & 19.303 \\
IP & IP-LPA & & & \\
HP & HP-IP & 0.311 & 0.025 & \\
\hline
\end{tabular}

\section{SSSC data:}

$$
\begin{aligned}
& K_{p}=10, \quad K_{I}=200, \\
& T_{\mathrm{sssc}}=0.1 \mathrm{sec}, \quad X_{\mathrm{ref}}=0.3 \mathrm{p} . \mathrm{u}, \\
& V_{d \text { cref }}=1 \mathrm{p} . \mathrm{u} .
\end{aligned}
$$

\section{Biographies}

Mahmoud Reza Shakarami was born in Khorramabad, Iran in 1972. In 2010, he received his $\mathrm{PhD}$ degree in Electrical Engineering from Iran University of Science and Technology in Tehran, Iran. He is currently an Assistant Professor in Electrical Engineering Department of Lorestan University, in Khorramabad, Iran. His research interests are power system dynamics and stability, FACTS devices, and distribution power systems.

Akbar Asadi Gheyasvand was born in Tehran, Iran in 1987. In 2015, he received his MS degree in Electrical Engineering from Lorestan University, in Khorramabad, Iran. His research interests are power system dynamics and stability and FACTS devices.

Ahad Kazemi was born in Tehran, Iran in 1952. He received his MS degree in Electrical Engineering from Oklahoma State University, USA in 1979. He is currently an Associate Professor in Electrical Engineering Department of Iran University of Science and Technology, in Tehran, Iran. His research interests are reactive power control, power system dynamics and stability, and FACTS devices. 